TECHNICAL NOTE

\author{
J. Ma \\ E.F. Jackson \\ A.J. Kumar \\ L.E. Ginsberg
}

\section{Improving Fat-Suppressed T2-Weighted Imaging of the Head and Neck with 2 Fast Spin-Echo Dixon Techniques: Initial Experiences}

\begin{abstract}
SUMmaRY: Two modified fast spin-echo (FSE) techniques (a 2-point and a single-scan triple-echo Dixon) were used for T2-weighted imaging of the head and neck in 7 patients along with conventional FSE with fat saturation. Both Dixon techniques provided consistent and more uniform fat suppression (FS) than conventional FSE. The 2-point Dixon technique was noted to be more susceptible to motion artifacts. The triple-echo Dixon technique offered the best scan time efficiency and overall image quality.
\end{abstract}

$\mathbf{F}^{a}$ t suppression (FS) is often required for T2-weighted imaging with the fast spin-echo (FSE) pulse sequence. ${ }^{1}$ However, magnetic field inhomogeneity induced by the anatomic geometry of the neck and the presence of many tissue-tissue and tissue-air interfaces in the head and face is difficult to compensate with hardware magnetic field shimming. As a result, the quality of FS in this anatomic region with use of the conventional chemical shift selective suppression (CHESS) technique is sometimes unsatisfactory. Multipoint Dixon techniques ${ }^{2,3}$ have the potential to provide better FS by overcoming field inhomogeneity effects with phase correction during image reconstruction. Successful applications of the 3-point FSE Dixon techniques have been reported. ${ }^{4-8}$ However, many 3-point FSE Dixon techniques increase the FSE echo spacing (which has a direct impact on the FSE image quality and the number of sections per TR) and increase the minimum total scan time; some are reported to have certain reconstruction errors. ${ }^{8}$ In this study, we applied an FSE 2-point Dixon (2PD) ${ }^{9}$ technique and a single-scan FSE triple echo Dixon technique (fTED) ${ }^{10}$ for fat-suppressed T2-weighting imaging of the head and neck in 7 patients with cancer. Compared with a 3-point Dixon technique, these 2 techniques require only either a 2-point acquisition ${ }^{9}$ or a single-scan acquisition. ${ }^{10}$ Both techniques also avoid using the often errorprone direct-phase unwrapping in postprocessing. Therefore, the 2 techniques have better scan time efficiency and processing reliability. ${ }^{9,10}$ We present our initial experiences in comparing their performance for T2-weighted head and neck imaging with that of the conventional FSE with CHESS fat suppression.

\section{Description of the Techniques}

For the 2PD technique, we used a modified FSE pulse sequence to acquire 2 interleaved input images (one in-phase and the other $180^{\circ}$ opposed-phase). ${ }^{9}$ We achieved the $180^{\circ}$ phase shift by increasing the echo spacing (the time interval between 2 successive $180^{\circ}$ radiofrequency refocusing pulses in the echo train) and by shifting the

Received October 29, 2007; accepted after revision February 6, 2008.

From the Departments of Imaging Physics (J.M., E.F.J.) and Diagnostic Radiology (A.J.K., L.E.G.), University of Texas M.D. Anderson Cancer Center, Houston, Tex.

One of the authors (J.M.) is a grant recipient of the Susan Komen Breast Cancer Research Foundation and the Bracco/Radiological Society of North America research scholar award.

Please address correspondence to Jingfei Ma, PhD, Department of Imaging Physics, University of Texas M.D. Anderson Cancer Center, Box 56, Houston, TX 77030; e-mail: jma@di.mdacc.tmc.edu

DOI 10.3174/ajnr.A1132

readout gradients/data acquisition window from the conventional spin-echo location. The amount of the shift and echo spacing increase are both proportional to the desired phase shift. Because 2 input images are acquired and the echo spacing is increased, the minimum total scan time of the 2PD technique is at least doubled relative to a conventional FSE scan with identical scan parameters. Nonetheless, the minimum total scan time of a $2 \mathrm{PD}$ acquisition is still reduced by at least one third relative to that of a 3-point Dixon acquisition. ${ }^{9}$

For the fTED technique, we acquired 3 input images in a single FSE scan by replacing each readout gradient in a conventional FSE with 3 consecutive gradients of alternating polarity. ${ }^{10} \mathrm{We}$ adjusted the timing of the 3 readout gradients by automatically selecting an appropriate receiver bandwidth so that the water and fat signals for the 3 corresponding echoes have a relative phase shift of $-180^{\circ}, 0$, and $180^{\circ}$, respectively. Ignoring the small amount of time needed for switching the gradients, the echo spacing and the minimum total scan time for fTED are unchanged when compared with the conventional FSE. Thus, fTED acquisition is substantially more efficient than the acquisition by any 3-point Dixon or even the $2 \mathrm{PD}$ techniques. For the image reconstruction, 2 separate sets of water-only and fat-only images are first generated for each section from the 2 images with $-180^{\circ}$ and 0 phase shifts, and the 2 images with 0 and $180^{\circ}$ phase shifts, respectively. These 2 sets of water-only and fat-only images are then combined for improved signal-to-noise ratio (SNR) in the final output images. ${ }^{10}$

Both the 2PD and fTED techniques used a recently published phase correction algorithm for water and fat separation. ${ }^{11}$ The algorithm and pulse sequences are not limited to a specific magnet type or vendor and have been successfully implemented at both $1.5 \mathrm{~T}$ and $3 \mathrm{~T}$ scanners. In contrast to many previously known 3-point Dixon techniques, the phase correction algorithm used in the $2 \mathrm{PD}$ and fTED techniques requires only 2 images (one with the water and fat inphase and the other with the 2 signals $180^{\circ}$ opposed-phase) and circumvents the need for error-prone direct-phase unwrapping. Instead, the algorithm uses a fully automated region-growing process to determine the phase vector distribution, which has been shown to be sufficient for water and fat separation. ${ }^{11}$ In more specific terms, the region-growing process uses 2 spatial gradients of the signal phase to guide an optimal growth sequence with no need for manual seed selection or use of an empiric angular threshold. Furthermore, the phase vector determination of a given pixel is based on both the amplitude and the phase vector of the surrounding pixels for which phase vectors have already been determined. Thus, the algorithm is highly robust in the presence of the pixels with large phase uncertainty and is very computationally efficient.

We developed and implemented in-house both the 2PD and the 

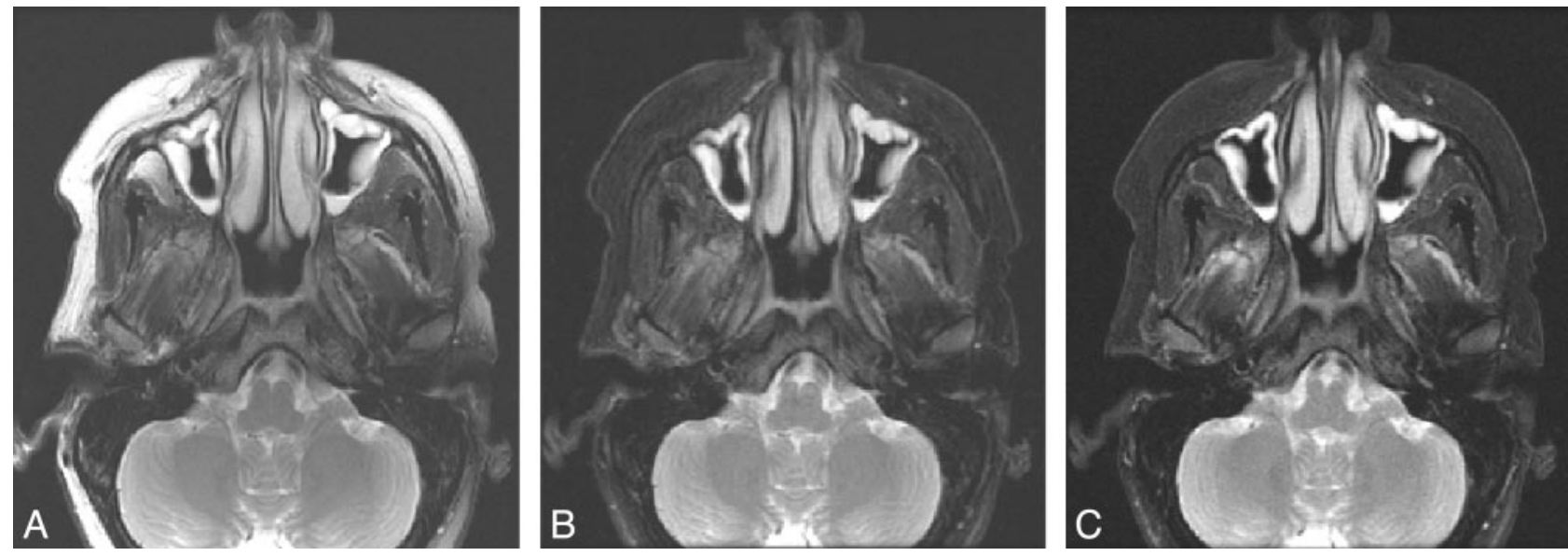

Fig 1. T2-weighted images obtained with a conventional FSE acquisition with CHESS $(A)$, the 2PD technique $(B)$, and the fTED technique (C). The images obtained with use of the 2 Dixon techniques provide uniform fat suppression throughout the FOV, whereas the fat suppression achieved by the conventional CHESS FSE technique is unsatisfactory in the anterior facial region.

fTED techniques as research prototype sequences on a $1.5 \mathrm{~T}$ Excite (GE Healthcare, Waukesha, Wis) whole-body scanner with $12 \mathrm{x}$ software. From March 8 to October 25, 2007, and within the constraints of scheduling flexibility and a radiologist's availability to obtain patient consent, we randomly enrolled 7 patients into this institutional review board-approved study from the patients who were referred for a routine head and neck MR imaging examination. The routine head and neck MR imaging protocol includes fat-suppressed T2-weighted imaging as well as T1-weighting imaging without fat suppression before and T1-weighted imaging with fat suppression after the injection of contrast agent. Among the 7 patients, 4 were men and 3 were women. The mean age of the patients was 59 years (range, 38-77 years). All study subjects had head and neck cancer (parotid, $n=2$; nasopharyngeal space, $n=2$; nasal/premaxillary, $n=1$; frontal sinus, $n=1$; and tongue, $n=1$ ). All patients provided written informed consent. From the 7 patients, we collected a total of 274 oblique axial sections, covering the lower skull and neck and cervical spine regions, using the 2 Dixon techniques as well as the conventional FSE technique with CHESS. An 8-channel neurovascular phased array coil was used for all scans. The conventional FSE with CHESS was part of the clinical protocol in use at our institution. A typical set of scan parameters was as follows: TR, $4800 \mathrm{~ms}$; TE (effective), $94 \mathrm{~ms}$; echo-train length (ETL), 10; receiver bandwidth (RBW), $\pm 15.6 \mathrm{kHz}$; acquisition matrix, $256 \times 256$; FOV, $16 \mathrm{~cm}$; section thickness/gap, 5/1.5 mm; NEX (number of excitations), 4; and total scan time, 8:24 minutes (for 28 sections). The scan parameters and section coverage for the 2 Dixon techniques were kept identical to those for the conventional FSE, except for the following changes: for the 2PD technique, TR, $5050 \mathrm{~ms}$; TE (effective), $90.4 \mathrm{~ms}$ (because of the increased echo spacing), RBW, $\pm 15.6 \mathrm{kHz}$; and total scan time, 8:50 minutes. For the fTED technique, TR, $4550 \mathrm{~ms}$; TE (effective), $96.2 \mathrm{~ms}$; RBW, \pm 62.5 $\mathrm{kHz}$ (to keep the total data readout time equivalent as the conventional FSE acquisition); and total scan time, 7:58 minutes. Compared with the conventional FSE acquisition, the slightly longer scan time for $2 \mathrm{PD}$ was the result of the increased echo spacing, and the slightly shorter scan time for fTED was because of the elimination of the CHESS pulses. For both Dixon techniques, image reconstruction and installation into the scanner image data base were automatic after the data acquisition. The image reconstruction time was approximately 1 second per image.

\section{Discussion}

Two neuroradiologists viewed the Dixon and the conventional FSE images side by side and did a subjective comparison for the study. The 2 Dixon techniques were found to achieve better and more uniform FS than the conventional FSE technique in all of the sections collected. In our clinical practice, manual prescan (including tuning of the center frequency and the optimal CHESS flip angle) was performed for T2-weighted imaging of all of the patients when needed. Despite these efforts, the FS quality was often found to be suboptimal. Figure 1 shows an example of the 3 images from the same anatomic location but acquired with the 3 different techniques. Failure of FS by CHESS is clearly noticeable in Fig $1 A$, particularly in the anterior facial region where the many air/tissue interfaces can make good field homogeneity difficult to achieve. In contrast, FS was uniform across the whole FOV for the images by the 2 Dixon techniques (Fig $1 B,-C$ ).

Excluding FS uniformity considerations, the image quality by the 3 different techniques was found to be mostly similar. However, we found that the $2 \mathrm{PD}$ technique is more susceptible to motion than the conventional FSE or the fTED techniques. Figure 2 shows another set of the images through the mouth and neck regions by the 3 different techniques. Although the 2 Dixon techniques again achieved much better FS than the conventional FSE with CHESS, ghosting artifacts can be seen in both the anterior and posterior portions of the image by the $2 \mathrm{PD}$ technique (as indicated by the arrows in Fig $2 B$ ). Although it needs to be confirmed in a study with a larger number of patients and more rigorous statistical analysis, we note that our observation on the motion susceptibility of the 2PD technique was consistent in all our patients. Figure 3 shows another example set of the images from a different patient. The window levels of the images were adjusted in a similar fashion to illustrate the motion artifacts more effectively. As in Fig 2, fat suppression in the 2 Dixon images (Fig 3B, $-C$ ) was more uniform than the image by CHESS (Fig $3 A$ ). On the other hand, motion artifacts (at the levels of the tongue and the carotid artery, as indicated by the 2 arrows) were most severe in the image by the $2 \mathrm{PD}$ technique. Furthermore, we note that these artifacts were present in the input images be- 

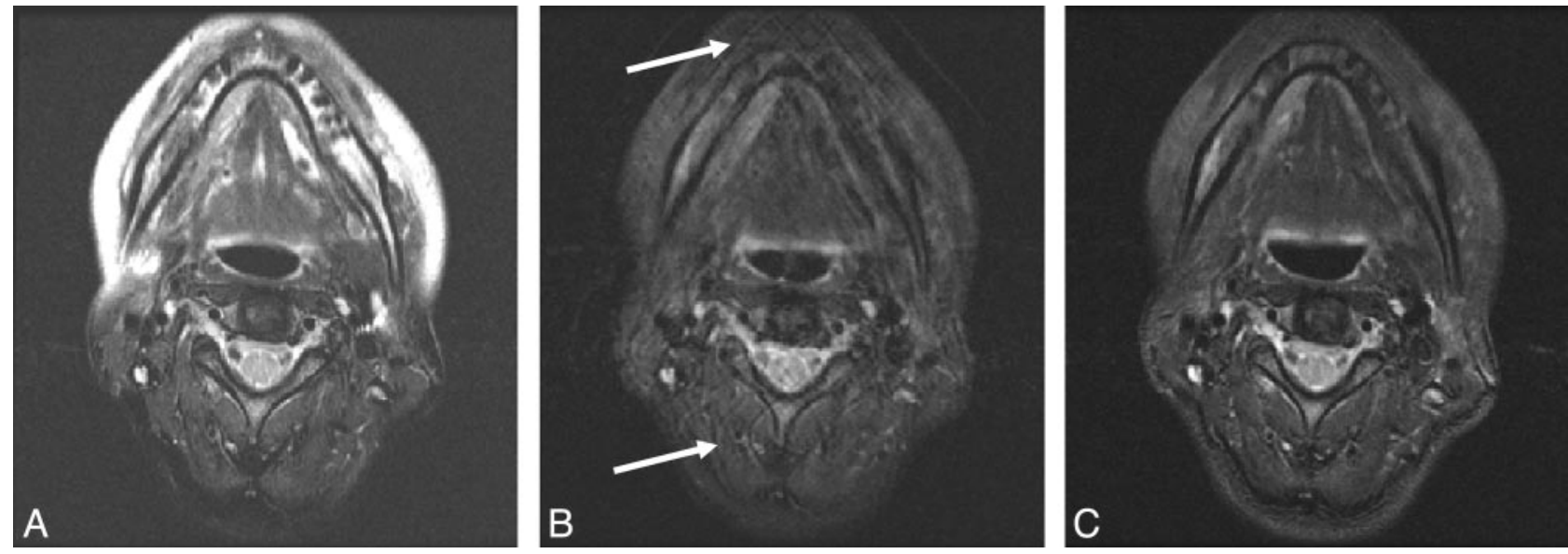

Fig 2. T2-weighted images obtained with a conventional FSE acquisition with CHESS $(A)$, the 2PD technique $(B)$, and the fTED technique $(C)$. The images obtained with use of the 2 Dixon techniques again provide subjectively better and more uniform fat suppression than the images obtained with the conventional CHESS FSE technique. However, motion artifacts are noted to be present in the images obtained with 2PD (arrows). In comparison, the fTED images show uniform fat suppression and best overall image quality.
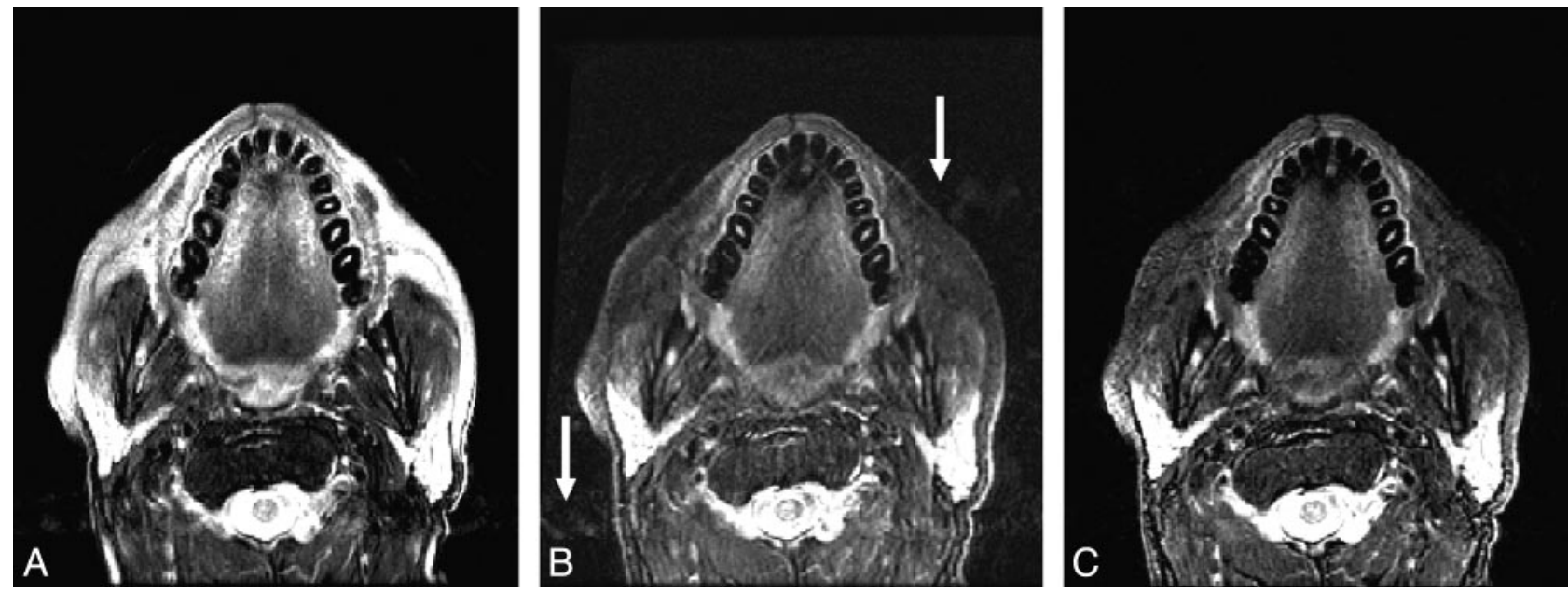

Fig 3. T2-weighted images of another patient obtained with a conventional FSE acquisition with CHESS $(A)$, the $2 P D$ technique $(B)$, and the fTED technique $(C)$. As in Fig 2 , fat suppression in the 2 Dixon images ( $B$ and $C$ ) was more uniform than that of the image by CHESS $(A)$. In contrast, motion artifacts (at the leve/s by the arrows) were most severe in the image by the 2PD technique.

fore the Dixon processing; thus, we attribute the root cause to a combination of involuntary motion (eg, mouth/throat and blood pulsation) and the interleaved acquisition used by the 2PD technique. Although no comparison was performed in this study, such sensitivity of motion is expected to be more aggravated for a 3-point Dixon technique with the increased number of interleaves. Another difference that is more subtle but clearly noticeable in the images in Figs 1-3, as well as all the other images not shown, is that the images by fTED demonstrate the best clarity and sharpness. We attribute this difference to the higher receiver bandwidth used in fTED, which reduces the $\mathrm{T} 2^{*}$ blurring during the data acquisition window.

Although only T2-weighted imaging was evaluated, we point out that the 2 Dixon techniques can also be used to acquire T1-weighted images with only slight changes in the scanning protocol. Such images are useful and are frequently acquired with the injection of gadolinium-based contrast agent. Because T1-weighted images usually require shorter acquisition time and have higher SNR, we expect equal or better reliability in processing than what was found for T2-weighted imaging. For all of the images evaluated in this study, we did not find any incorrect water and fat separation or processing dependence on prescan parameter settings, which can be another advantage compared with a recent implementation of the 3 -point Dixon technique. ${ }^{8}$

For our study, the total scan time for each of the 3 different techniques was kept similar to achieve similar overall SNR. However, the minimum possible total scan time of the 2PD technique was more than double that for the conventional FSE or fTED because of its requirement for a minimum of 2 NEX acquisition and increased echo spacing. We note that the minimum possible total scan time is further increased with an implementation of any 3-point Dixon techniques. ${ }^{4-8}$ As a result, the 2PD and 3-point Dixon techniques may be limited in the flexibility of choosing the scanning parameters. In contrast, the fTED implementation achieves the single scan efficiency and requires less compromise in scan protocols compared with the conventional FSE.

In summary, we found in our preliminary study that both 2PD and fTED techniques provided consistent and much more uniform FS than the conventional FSE with CHESS in T2-weighted imaging of the head and neck. The 2PD tech- 
nique was noted to be more susceptible to motion artifacts than the conventional FSE with CHESS or the fTED techniques. In general, the fTED technique achieved the best scan time efficiency and overall image quality. The fTED technique also requires the shortest minimum scan time of the $2 \mathrm{PD}$ or any other 3-point Dixon techniques. Thus, the fTED technique may offer the best alternative to conventional fat-suppressed FSE acquisitions for T2-weighted imaging of the head and neck.

\section{References}

1. Hennig J, Nauerth A, Friedburg H. RARE imaging: a fast imaging method for clinical MR. Magn Reson Med 1986;3:823-33

2. Dixon WT. Simple proton spectroscopic imaging. Radiology 1984;153:189-94

3. Glover GH, Schneider E. Three-point Dixon technique for true water/fat decomposition with B0 inhomogeneity correction. Magn Reson Med 1991;18:371-83

4. Hardy PA, Hinks RS, Tkach JA. Separation of fat and water in fast spin-echo
MR imaging with the three-point Dixon technique. J Magn Reson Imaging 1995;5:181-85

5. Rybicki FJ, Mulkern RV, Robertson RL, et al. Fast three-point Dixon MR imaging of the retrobulbar space with low-resolution images for phase correction: comparison with fast spin-echo inversion recovery imaging. $A J N R$ Am J Neuroradiol 2001;22:1798-802

6. Ma J, Singh SK, Kumar AJ, et al. T2-weighted spine imaging with a fast threepoint Dixon technique: comparison with chemical shift selective fat suppression. J Magn Reson Imaging 2004;20:1025-29

7. Reeder SB, Wen Z, Yu H, et al. Multicoil Dixon chemical species separation with an iterative least-squares estimation method. Magn Reson Med 2004;51:35-45

8. Barger AV, DeLone DR, Bernstein MA, et al. Fat signal suppression in head and neck imaging using fast spin-echo-IDEAL technique. AJNR Am J Neuroradiol 2006;27:1292-94

9. Ma J, Son JB, Bankson JA, et al. A fast spin echo two-point Dixon technique and its combination with sensitivity encoding for efficient T2-weighted imaging. Magn Reson Imaging 2005;23:977-82

10. Ma J, Son JB, Zhou Y, et al. Fast spin-echo triple-echo Dixon (fTED) technique for efficient T2-weighted water and fat imaging. Magn Reson Med 2007;58:103-09

11. Ma J. Breath-hold water and fat imaging using a dual-echo two-point Dixon technique with an efficient and robust phase-correction algorithm. Magn Reson Med 2004;52:415-19 